Rapid Communications

\title{
Tracking the Growth of Tadpole-shaped Aggregates by Scanning Electron Microscopy
}

\author{
Takamasa Kinoshita,* Hiroya Murakami,* Yusuke Muranaka,* Yojiro Yamamoto,*,** \\ Tomoaki Nishino, ${ }^{*}$ Hiroshi SHIIGI, ${ }^{* \dagger}$ and Tsutomu NAGAOKA* \\ *Department of Applied Chemistry, Osaka Prefecture University, 1-2 Gakuen, Sakai, Osaka 599-8570, Japan \\ **GreenChem Inc., 930-1 Fukuda, Sakai, Osaka 599-8241, Japan
}

\begin{abstract}
A single-step strategy has been developed toward the synthesis of micrometer-sized, tadpole-like structures comprising a repeating sequence of a three-dimensional gold nanoparticle (AuNP)-aniline polymer-AuNP arrangement. The size and shape of the microstructure strongly depends on the concentration of aniline in the reaction solution. Herein, we describe the formation mechanism of the microstructure by focusing on the surface morphology.
\end{abstract}

Keywords Gold nanoparticle, organic-inorganic hybrid, molecular bridging, scanning electron microscopy

(Received January 11, 2014; Accepted January 20, 2014; Published March 10, 2014)

Metal nanoparticles (NPs) have unique electronic and optical properties, and their applications in emerging areas of nanotechnology have made them topics of fundamental interest to many researchers. ${ }^{1-7}$ NPs have various shapes and aggregated structures, including barcodes, rods, and wires, which result in unique functions in addition to some rare functions of bulk metals. ${ }^{8-10}$ The construction of functional architectures and the facilitation of particle handling is troublesome because of the necessity of multistep processing. Previously, gold NPs (AuNPs) were synthesized at the aqueous/organic solution interface, and then covered with thiol molecules in organic media. ${ }^{11-15}$ The thiol passivation layer was then exchanged or modified using a solution of functional compounds for over $10 \mathrm{~h}$. This layer, however, only provided low-density coverage of functional molecules on the surface of the AuNPs, and the functionalization was therefore insufficient for the further construction of functional architectures.

To construct superstructures with AuNPs as functional accessories, it is necessary to develop the desired size and shape under specific reaction conditions. We have reported a simple method for preparing nanometer-sized hybrids consisting of AuNPs and aniline oligomers that does not require any extra step for reactivity control, organic solvents, or ligand exchange. ${ }^{16}$ In this paper, we describe a procedure to prepare simple tadpole-like structures using AuNP as a building block. The size and shape of the microstructure strongly depends on the concentration of aniline in the reaction solution. We monitored the surface morphology by scanning electron microscopy (SEM) and determined the formation mechanism of the microstructure.

The simple procedure to prepare the structures is as follows: Aqueous solutions of aniline (Wako Pure Chemical Industries, Japan), with concentrations from 2.0 to $87 \mathrm{mM}$, were added to an aqueous solution of $24 \mathrm{mM}$ chloroaurate (Tanaka Kikinzoku Kogyo, Japan). After the mixture was stirred at a constant speed

$\dagger$ To whom correspondence should be addressed.

E-mail: shii@chem.osakafu-u.ac.jp of $370 \mathrm{rpm}$ at $60^{\circ} \mathrm{C}$ for $30 \mathrm{~min}$, the resulting dispersions were centrifuged at $8500 \mathrm{rpm}$ for $20 \mathrm{~min}$ at $5^{\circ} \mathrm{C}$. The precipitate was added to $10 \mathrm{~mL}$ of ultrapure water, and then dispersed again by ultrasonication at $28 \mathrm{kHz}$ and $100 \mathrm{~W}$ for $1 \mathrm{~min}$. The dispersion was centrifuged under the same conditions as described above. After this procedure was repeated three times, the resulting dispersion contained no unreacted species. The obtained microstructures were characterized by field emission-scanning electron microscopy (SEM, S-4700, Hitachi, Japan) with an accelerating voltage of $200 \mathrm{kV}$.

The SEM images showed distinctly different shapes, depending on the concentration of aniline in the sample. A homogeneous raspberry-like hybrid, prepared by stirring the mixture solution after adding $2.0 \mathrm{mM}$ of aniline to $24 \mathrm{mM}$ of chloroaurate, was obtained with a mean diameter of $c a .150 \mathrm{~nm}$, as shown in Fig. 1a. The hybrid possessed numerous AuNPs with a mean diameter of $c a .5 .0 \mathrm{~nm} \cdot{ }^{17}$ Image (b) also shows the formation of a homogeneous hybrid. However, the hybrid possessed a broad particle size distribution with mean diameters of from 100 to $500 \mathrm{~nm}$. These results were in good agreement with a previous report, in that the diameter of the hybrid strongly depended on the reaction conditions. ${ }^{16}$ On the other hand, a heterogeneous, mushroom-shaped aggregate was also observed (Fig. 1c). A significant growth of tails was observed as the concentration of aniline increased (Figs. 1d-1g). Finally, tadpole-shaped aggregates were obtained at concentrations of aniline over $87 \mathrm{mM}$, as shown in Fig. 1h. Such shapes were observed previously for structures obtained in an organic/aqueous biphasic mixture. ${ }^{18}$ The minuscule organic droplets in the water functioned as minute reaction fields in the aqueous medium due to stirring of the solution. These fields supported aggregation with uniform shapes. In aqueous media with concentrated aniline, the minuscule droplets formed by the aniline in the water acted as minute reaction fields (the solubility of aniline is $390 \mathrm{mM}$ at $293 \mathrm{~K}$ ), as shown in Scheme 1. The aurate was reduced by the aniline to AuNPs, and the aniline monomer was oxidized via the self-stabilized dispersion polymerization 

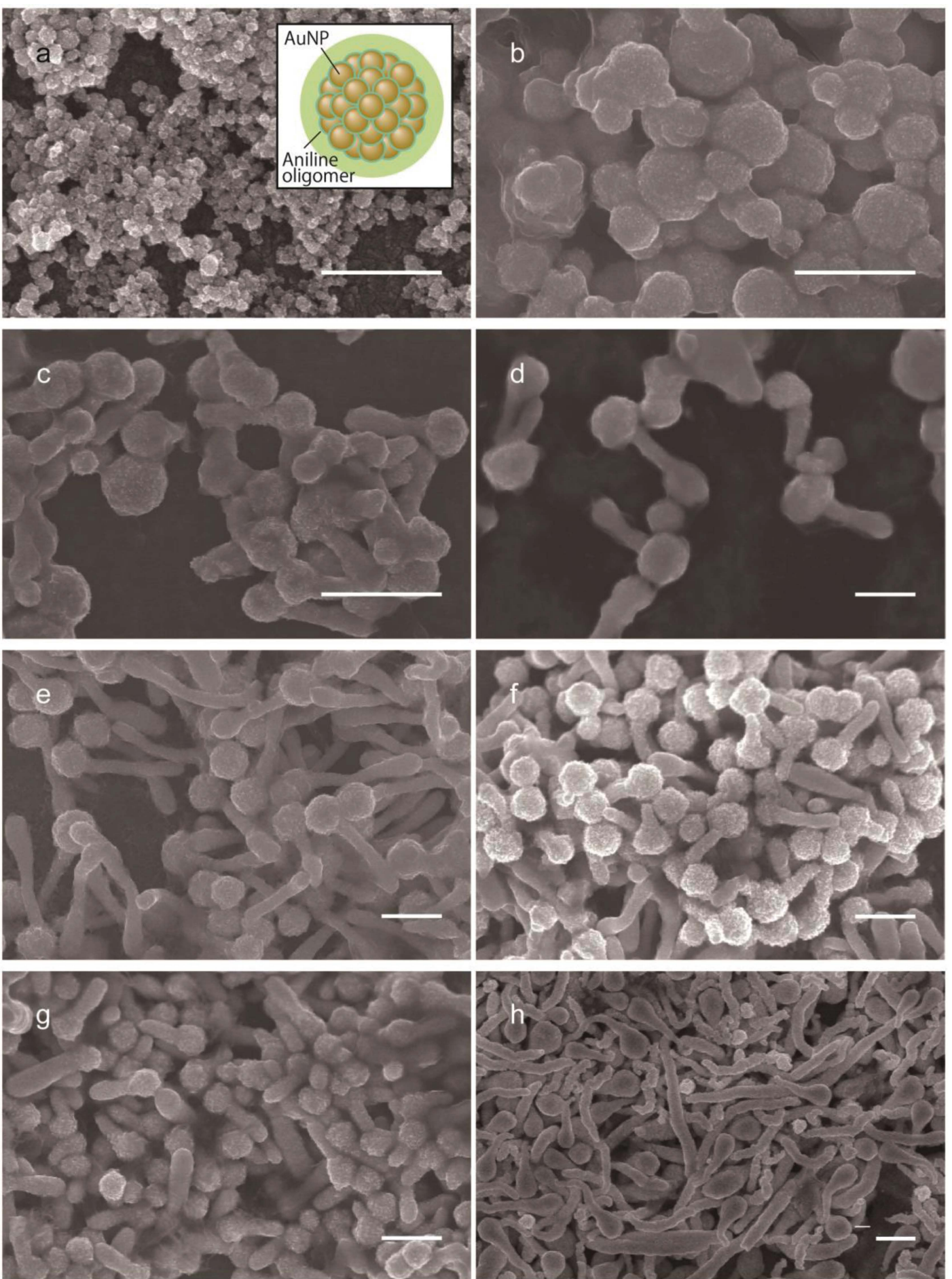

Fig. 1 SEM images of the raspberry-like hybrid (a) and microstructures $(b-h)$ prepared in solutions containing 2.0 (a), 54 (b), 77 (c), 80 (d), 82 (e), 84 (f), 86 (g), and 87 (h) $\mathrm{mM}$ aniline and $24.3 \mathrm{mM}$ of chloroaurate. Scale bar: $1 \mu \mathrm{m}$.

reaction in the heterogeneous biphasic mixture comprised of the aniline and water phases. ${ }^{19}$ The oxidation of the aniline proceeded outward radially, which presumably promoted successive para-mode coupling of the benzene rings of the aniline monomers as they diffused away from the reactive front. The radicals generated in the aqueous phase initiated polymerization on the surface of the aniline droplets, ${ }^{20}$ and radially assembled AuNPs were formed (Figs. 1a and 1b). The radial hybrids then diffused from the reaction field to the aqueous phase (Fig. 1c). Simultaneously, the hybrid grew and the tail increased in length from the interface to the aqueous phase (Figs. 1d-1g). Finally, mature tadpole-shaped aggregates were obtained, as shown in Fig. 1h. Therefore, it seems that the length of the tail of the microstructure depends on the size of the aniline droplet based on the concentration of aniline in the reaction solution.

To investigate the mechanism by which the aggregate formed, spectroscopic measurements were carried out. The UV-vis 


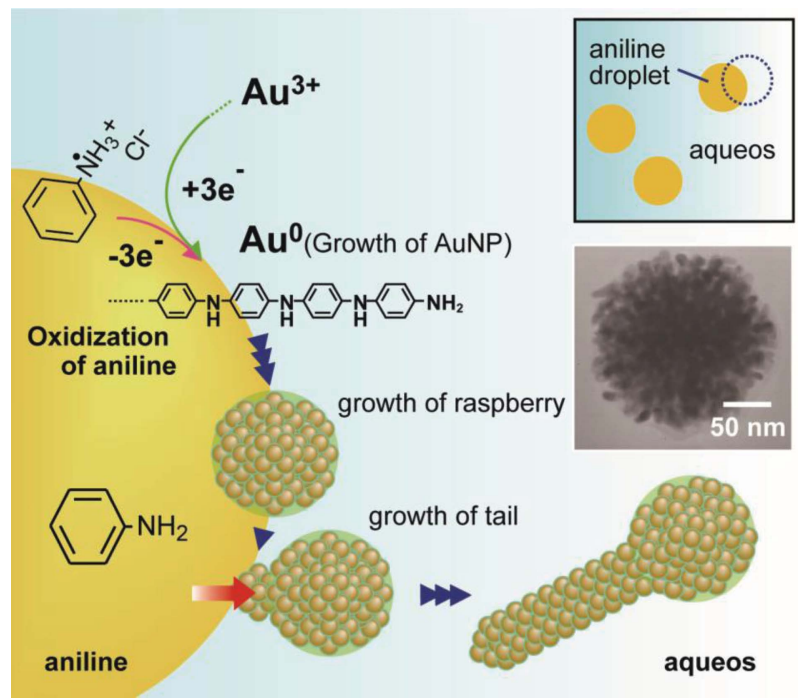

Scheme 1 Model illustration of the formation of a tadpole-like microstructure. a) raspberry

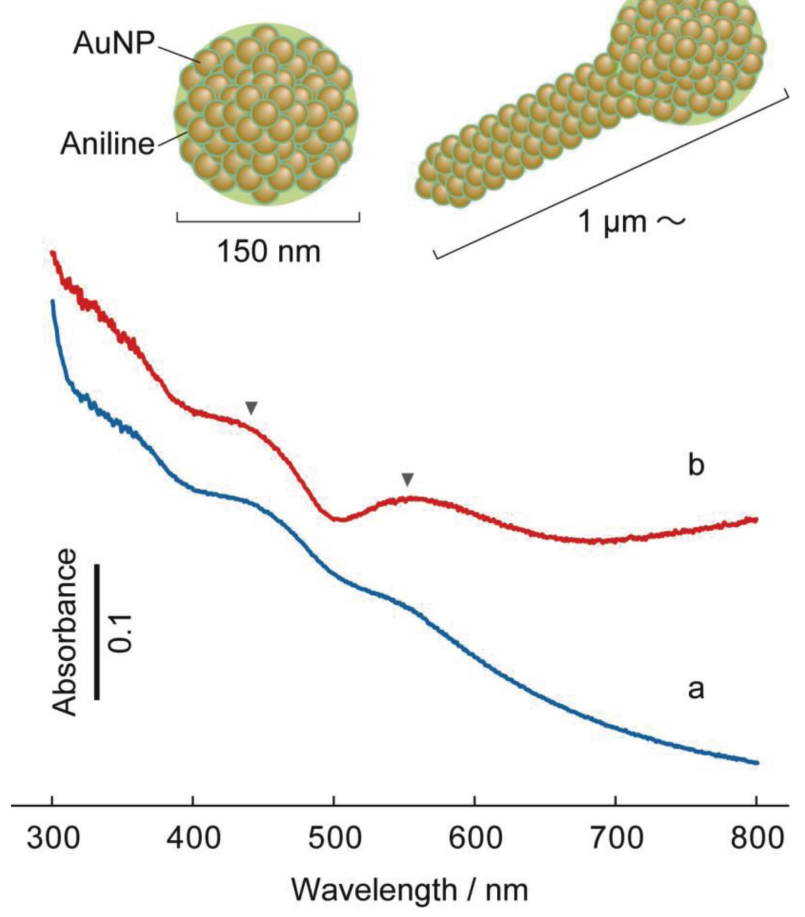

Fig. 2 UV-vis spectra of the raspberry-like hybrid (a) and the tadpole-like microstructure (b).

spectrum of the raspberry-like hybrid had significant peaks at 450 and $560 \mathrm{~nm}$, which could be attributed to the generation of a localized polaron (cation radical) of polyaniline (PANI) ${ }^{21-25}$ and the surface plasmon resonance (SPR) of the AuNP, respectively (Fig. 2a). ${ }^{16}$ The SPR of the hybrid was clearly observed, although it shifted to a wavelength about $40 \mathrm{~nm}$ longer than that typically reported for AuNPs because of coupling of the SPRs caused by the close packing of AuNPs. ${ }^{26}$ In the spectrum of the tadpole aggregate shown in Fig. 2b, absorption at wavelengths longer than $700 \mathrm{~nm}$ was generated, in addition to the peaks at 450 and $560 \mathrm{~nm}$. There are two potential explanations for the absorbance characteristics at long wavelength. One is based on the occurrence of an electronic interaction between the band structure of PANI and the surface electric fields of the AuNPs. The other explanation is based on the presence of a delocalized polaron in the long chains of PANI. Auric acid acts as a moderate oxidant (standard potential: $+1.52 \mathrm{~V}$ ), and therefore the polymerization of aniline proceeds along with the growth of $\mathrm{Au}^{0}$ on the AuNPs through the reduction of aurate..$^{27,28}$ On the other hand, it is expected that the formation of long chains should not be promoted in the weakly acidic aqueous phase ( $\mathrm{pH} 4)$. The aniline monomer was chemically oxidized by $\mathrm{Au}^{3+}$, which led to the formation of oligomers. These results were in good agreement with a previous report in regard to the shape-dependent electrochemical characteristics of the aggregates. ${ }^{17}$ The microstructure composed of the raspberry-like hybrid showed a distinctly different electrochemical response, as compared to that of the dispersed raspberry, and was very similar to the typical voltammogram of the conducting form of PANI. This meant that the aniline oligomers, which acted as a passivation layer for the raspberry-like hybrid, overlapped through $\pi$-stacking of the aromatic rings in the oligomers between the adjacent raspberries in the microstructure. However, the tadpole microstructures have indicated a good shape stability for over one month, since there is hardly any generation of raspberry due to decomposition from the aggregation observed in the dispersion. ${ }^{17}$ This indicates that tadpoles have not been formed through the weak interactions between the raspberries as building blocks. In concentrated aniline solutions, it is expected that the aniline monomers supplied endlessly to the reaction fields, which correspond to the surface of the droplet, are oxidized after the other by auric acids diffusing from the aqueous phase. That the oxidation of aniline proceeds continuously allows for the formation of the long chain (or PANI), even in a weak acid solution. Therefore, the tadpole microstructure possesses good shape stability in contrast to that found in previous work. ${ }^{16,17,25}$

In summary, we successfully prepared tadpole-shaped microstructures in a single step by controlling the concentration of aniline, which acts as a reducer and passivation layer after the formation of aggregates. A comparison between the spectroscopic and electrochemical characteristics of the raspberry-like hybrid and the tadpole aggregate revealed a significant difference based on the aggregation states responsible for their shapes and sizes. In dilute solutions of aniline, a homogeneous reaction occurs in the numerous minuscule reaction fields. In concentrated solutions of aniline, the reactions also occur homogeneously on the surface of aniline droplets, and proceed the polymerization into long chains even in a weak acid solution. Therefore, the tadpole-shaped aggregates indicated good stability.

\section{Acknowledgements}

We gratefully acknowledge financial support provided by the Ministry of Agriculture, Forestry, and Fisheries through a Science and Technology Research Promotion Program for Agriculture, Forestry, Fisheries and Food Industry; and the Japan Society for the Promotion of Science through a Grant-in-Aid for Scientific Research (B) (JSPS KAKENHI, 25288039, 25288069). 


\section{References}

1. H. Shiigi, S. Tokonami, Y. Yamamoto, and T. Nagaoka, Anal. Sci., 2012, 28, 1037.

2. K. C. Grabar, R. G. Freeman, M. B. Hommer, and M. J. Natan, Anal. Chem., 1995, 67, 735.

3. J. J. Storhoff, A. A. Lazaorides, R. C. Mucic, C. A. Mirkin, R. L. Letsinger, and G. C. Schatz, J. Am. Chem. Soc., 2000, $122,4640$.

4. K. Sato, K. Hosokawa, and M. Maeda, J. Am. Chem. Soc., 2003, 125, 8102 .

5. Y. Kubo, S. Uchida, Y. Kemmochi, and T. Okubo, Tetrahedron Lett., 2005, 46, 4369.

6. I. D. Walton, S. M. Norton, A. Balasingham, L. He, D. F. Oviso, D. Gupta, P. A. Raju, M. J. Natan, and R. G. Freeman, Anal. Chem., 2002, 74, 2240.

7. C.-C. Chen, Y.-P. Lin, C.-W. Wang, H.-C. Tzeng, C.-H. Wu, Y.-C. Chen, C.-P. Chen, L.-C. Chen, and Y.-C. Wu, J. Am. Chem. Soc., 2006, 128, 3709.

8. C. A. Foss, G. L. Hornyak, J. A. Stockert, and C. R. Martin, J. Phys. Chem., 1994, 98, 2963.

9. L. Li and S. I. Stupp, Angew. Chem., 2005, 44, 1833.

10. H. Nakao, S. Tokonami, T. Hamada, H. Shiigi, T. Nagaoka, F. Iwata, and Y. Takeda, Nanoscale, 2012, 4, 6814.

11. I. Hussain, Z. Wang, A. I. Cooper, and M. Brust, Langmuir, 2006, 22, 2938.

12. M. G. Warnerd and J. E. Hutchison, Nat. Mater, 2003, 2, 272.

13. T. Yonezawa, S. Onoue, and S. Kimizuka, Chem. Lett., 2002, 1172.

14. C. M. McIntosh, E. A. Esposito, A. K. Boal, J. K. Simard,
C. T. Martin, and V. M. Rottelo, J. Am. Chem. Soc., 2001, 123, 7626.

15. V. J. Gandubert and R. B. Lennox, Langmuir, 2005, 21, 6532.

16. H. Shiigi, Y. Yamamoto, N. Yoshi, H. Nakao, and T. Nagaoka, Chem. Commun., 2006, 4288.

17. H. Shiigi, Y. Muranaka, Y. Yamamoto, and T. Nagaoka, Anal. Sci., 2013, 29, 937.

18. H. Shiigi, R. Morita, Y. Yamamoto, S. Tokonami, H. Nakao, and T. Nagaoka, Chem. Commun., 2009, 3615.

19. K. Lee, S. Cho, S. H. Park, A. J. Heeger, C.-W. Lee, and S.-H. Lee, Nature, 2006, 441, 65.

20. J. Huang and R. B. Kaner, J. Am. Chem. Soc., 2004, 126, 851.

21. A. A. Nekrasov, V. F. Ivanov, and A. V. Vannikov, J. Electroanal. Chem., 2000, 482, 11.

22. K. Ogura, R. C. Patil, H. Shiigi, T. Tonosaki, and M. Nakayama, J. Polym. Sci., Part A: Polym. Chem., 2000, 38, 4343.

23. J. M. Kinyanjui, D. W. Hatchett, J. A. Smith, and M. Josowicz, Chem. Mater, 2004, 16, 3390.

24. J. M. Kinyanjui, J. Hanks, D. W. Hatchett, A. Smith, and M. Josowicz, J. Electrochem. Soc., 2004, 151, D113.

25. H. Shiigi and T. Nagaoka, Anal. Sci., 2014, 30, 89.

26. J. Gao, M. C. Bender, and J. C. Murphy, Langmuir, 2003, 19, 9065.

27. A. J. Bard, R. Parsons, and J. Jordan, "Standard Potentials in Aqueous Solution", 1985, Marcel Dekker, New York, 795.

28. Z. Guo, Y. Zhang, L. Haung, M. Wang, J. Wang, J. Sun, L. $\mathrm{Xu}$, and N. Gu, J. Colloid Interface Sci., 2007, 309, 518. 